\title{
Bilateral Choroidal Metastases In Triple-Negative Breast Cancer: Radiotherapy And Eribulin For Vision Preservation And Prolong Survival
}

\author{
Harissa H Hasbullah ${ }^{1,3^{*}}$, Zulia Zulkiffli ${ }^{2}$, Han Albert ${ }^{3}$ \\ ${ }^{1}$ Oncology Unit, Faculty of Medicine, UiTM Sg Buloh, Selangor, Malaysia \\ ${ }^{2}$ Department of Medicine, Hospital Tanah Merah, Kelantan, Malaysia \\ ${ }^{3}$ Department of Oncology and Radiotherapy, General Hospital Kuala Lumpur, Malaysia
}

\section{ARTICLE INFO}

Received : 04 June 2021

Reviewed : 19 July 2021

Accepted : 30 August 2021

Keywords:

choroidal metastases, eribulin,

triple-negative breast cancer
*Corresponding author:

Harissa Husainy Hasbullah

Oncology Unit, Faculty of Medicine,

UiTM Selangor, Jalan Hospital,

47000 Selangor, Malaysia

harissa@uitm.edu.my

\begin{abstract}
Introduction: Choroidal metastases are infrequent in breast cancer, but if they present, they usually signify the disseminated disease and poor prognosis. The challenges in treating choroidal metastases are not only to prolong survival but also to preserve vision, improving the quality of life.
\end{abstract}

Case Presentation: Our patient was firstly diagnosed with stage-three triple-negative breast cancer at the age of 32 years. She had surgery, adjuvant chemotherapy with anthracycline regime, as well as adjuvant radiotherapy. Her disease firstly recurred two years later with pleural effusion, but it was controlled with six cycles of docetaxel. She was in remission until ten years later when she presented with a worsening dry cough and progressive blurring of vision in both eyes. CT restaging showed multiple sub-centimeter bilateral lung nodules, singular pleural metastases, and multiple bone metastases. Choroidal metastases were also confirmed with the ophthalmological assessment which includes $\mathrm{CT}$ of the orbit. She received short-course palliative radiotherapy followed immediately by eribulin. Then, she started monthly bisphosphonates. She was able to read again four months after radiotherapy, and her vision remains normal to date. The latest PET scans showed no FDG avid disease in the lungs with pleural metastases significantly reduced in size. Bone metastases remain stable and asymptomatic. It has been nearly four years since the diagnosis of choroidal metastases. She is still on eribulin at an adjusted dose and interval. She remains asymptomatic from her bone, lung, and choroidal metastases.

Conclusions: Short-course radiotherapy to the orbit, followed by continuous administration of eribulin, can lead to prolonged survival with a good quality of life in triple-negative breast cancer with choroidal metastases.

\section{INTRODUCTION}

Breast cancer is the most common cancer among women in the world and in many countries including Malaysia. Unfortunately, in Malaysia, about one out of four breast cancer patients presented with stage four when the survival rate was only $23.3 \%$ at 5 years with the worst survival in triple-negative patients [1,2].

The most common sites of metastases are bone, lung, liver, and brain. Choroidal metastasis is uncommon, and, when present, it usually signifies the disseminated disease and poor prognosis. Median survival for breast cancer with choroidal metastases has been reported to be between 9 to 22 months [3].
There have been numerous case reports and case series published on choroidal metastases. However, most of them are of either hormonal positive or HER2 amplified breast cancer patients [4] whose prognosis and systemic treatments differ from triple-negative breast cancer patients. Meanwhile, of the choroidal metastases cases specifically reported in triple-negative breast cancer, survival was limited to less than a year [5].

We believe that our patient is the firstly reported triple-negative breast cancer patient with choroidal metastases who are enjoying a long survival of almost four years to date by using a combination of radiotherapy and maintenance eribulin. 


\section{CASE PRESENTATION}

MA was initially diagnosed with stage III (T3N1MO) triple-negative infiltrating ductal carcinoma (IDC) of the breast at the age of 32 years. She had no known comorbid and no risk factors for breast cancer. Right mastectomy and axillary clearance were done in August 2005 followed by six cycles of adjuvant chemotherapy with 5-fluorouracil, anthracycline, and cyclophosphamide. She also had adjuvant radiotherapy to the right chest wall and right supraclavicular fossa area completed by April 2006.

She was under regular follow-up at the oncology clinic, but unfortunately, her disease recurred in September 2007 when she presented with right pleural effusion. She was given six cycles of palliative chemotherapy docetaxel which was completed in January 2008. Surveillance CT in June 2008 showed minimal residual pleural effusion but no lung nodules nor disease elsewhere.

She continued to be under regular follow-up at our clinic and was in remission until July 2017 when she presented with a month-long history of worsening cough associated with lethargy. CT TAP July 2017 showed multiple sub-centimeter bilateral lung nodules with pleural metastases at the posterior right lung measuring $4.4 \mathrm{~cm}$ (Figure 1A). There were also matted nodes at the right superior mediastinum, right hilum, and enlarged nodes at subcarinal and precarina (Figure 1B) as well as bone metastases at T3, T9, left $9^{\text {th }}$ ribs, T10, and T12.
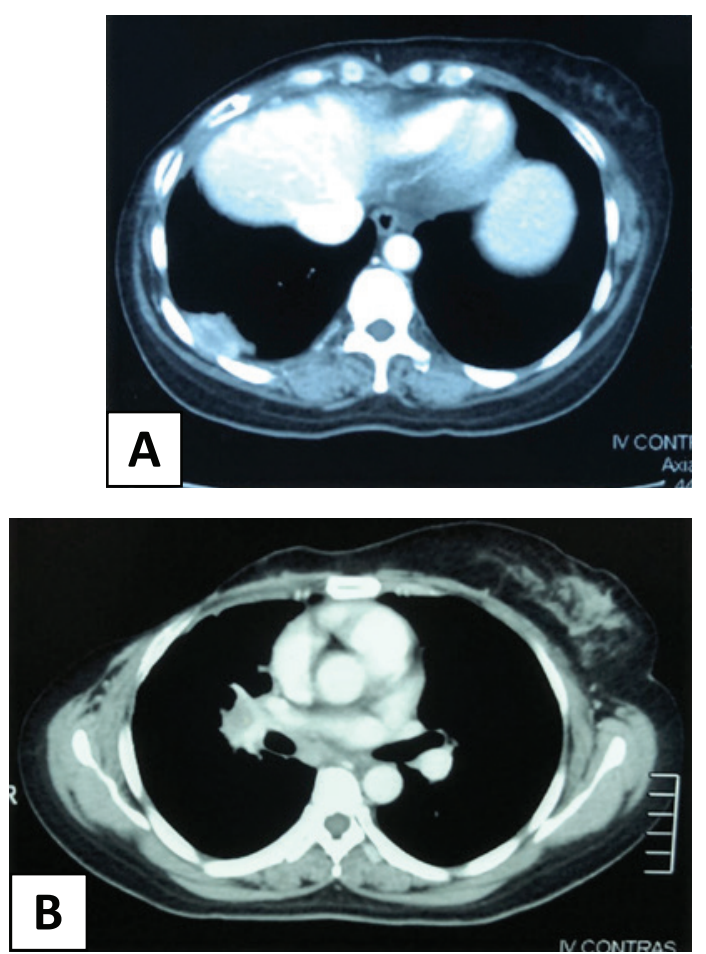

Figure 1. CT scan July 2017. (A) Pleural metastases at the posterior right lung of $4.4 \mathrm{~cm}$; (B) Matted nodes at the right hilum.
Unfortunately, prior to starting third-line palliative chemotherapy, the patient developed progressive blurring of her eyes. She was seen by an ophthalmologist who noted bilateral optic disc swelling possibly due to external compression of the optic nerves. A CT of the orbit showed features consistent with possible bilateral choroidal metastases (Figure 2A).

Radiotherapy of 20 Gy fractionated over 5 days (one week) using external beam radiotherapy (EBRT) with $6 \mathrm{MV}$ photon from a linear accelerator (LINAC) was delivered as laterally opposed beams targeting the bilateral choroidal metastases. Two weeks after completing the radiotherapy, she started intravenous eribulin $1.4 \mathrm{mg} / \mathrm{m}^{2} \mathrm{D} 1, \mathrm{D} 8$ every three weeks. Cycle one started on August 15, 2017.
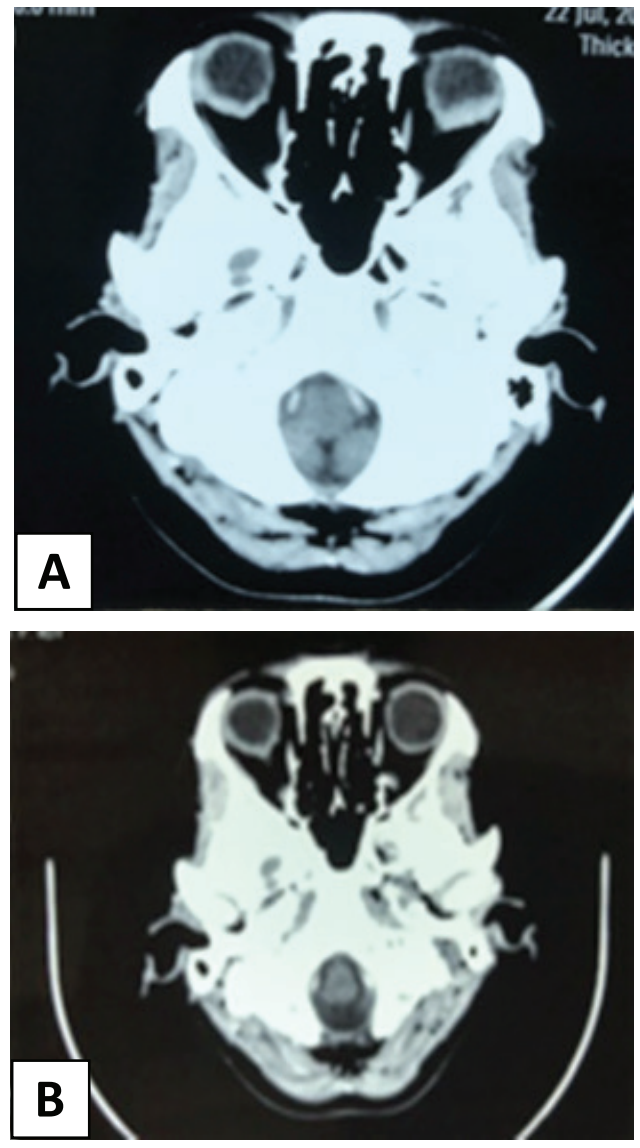

Figure 2.

Choroidal

metastases on

CT images

(A) At diagnosis (July 2017);

(B) 10 months after radiotherapy (June 2018);

(C) complete resolution of bilateral choroidal metastases 15 months after radiotherapy (November 2018)

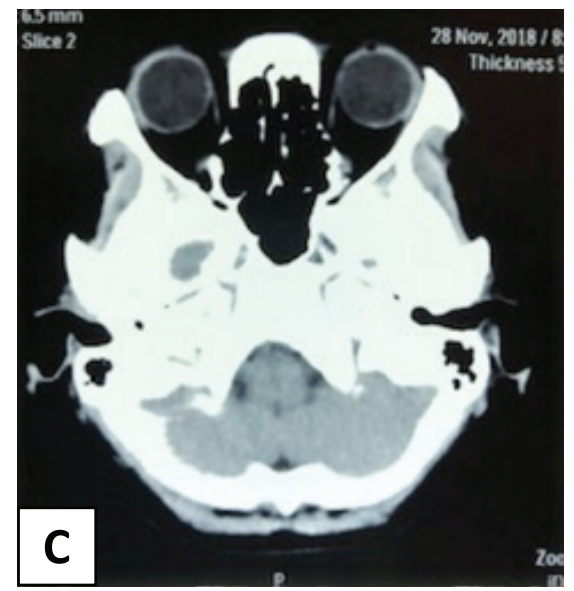


Her vision started to show improvement at about three months post-radiotherapy. By December 2017, she was able to read again. Upon her visit for the $11^{\text {th }}$ cycle of eribulin on April 24, 2018, MA claimed her vision was completely back to normal. CT reassessment in June 2018 after 13 cycles of eribulin showed an improvement of the choroidal metastases (Figure 2B). The pleural-based plaque has also been reduced in size to $0.9 \mathrm{~cm}$. The right hilar node now is only $0.8 \mathrm{~cm}$. All the matted superior mediastinal nodes and other mediastinal nodes had disappeared. The sub-centimeter lung nodules had reduced remarkably both in number and sizes. The $\mathrm{CT}$, however, reported a new bone lesion in T11, L3, L4, and pelvic bones although the patient remained asymptomatic from them. Because of the new bone metastases, monthly zoledronic acid was added, but eribulin was continued because of a good response elsewhere.

A repeated CT scan on November 28, 2018, after 19 cycles of eribulin, continued to show stable pleural plaque, lung nodules, bone disease, and sub-centimeter hilar nodes. Choroidal metastases have completely resolved (Figure $\mathbf{2 C}$ ).

It has been nearly four years since she had the diagnosis of choroidal metastases and just had her cycle 49 on day 1 eribulin on June 3, 2021; she remained well and asymptomatic of her underlying disease. Her vision remained good with no toxicity experience from the previous orbit irradiation. She continued to enjoy teaching and an active lifestyle. She recently enjoyed a month of a break from treatment in the month of Ramadhan.

The most recent imaging is a PET scan in July 2020 which showed FDG avid disease only in the sternum, T9, T11, and T12 with an SUV max of 4.0. There was a slightly lower SUV max in level I axillary lymph node although this was impalpable clinically and not seen on the serial CT scan previously.

During her first year of treatment with eribulin (from August 2017 until November 2018), she developed two separate incidents of grade- 2 afebrile neutropenia which recovered to normal after an a-week delay in treatment on both occasions. She also had grade-I peripheral neuropathy which persisted until today although lately, she has claimed that there has been improvement, and it does not hinder her daily activity.

During her second year of treatment with eribulin (December 2018 until December 2019), she had three separate episodes of afebrile neutropenia of grades 2 and 3 which again recovered following an a-week delay in treatment. She also had one episode of grade-I liver transaminitis which resolved upon stopping the use of an over-the-counter (OTC) supplement of cow's milk colostrum.

In 2020 (the third year of treatment), the patient remained clinically well. However, her neutropenia was getting longer to recover, and she even experienced one episode of grade-4 neutropenia. We had to adjust her eribulin regime several times during the third and fourth years of treatment. Her final regime to date is $1.1 \mathrm{mg} / \mathrm{m}^{2} \mathrm{D} 1$ and D15 every 28 days. With this regime so far, she has had two episodes of grade-2 afebrile neutropenia which fully recovered following a week of rest.

Despite these toxicities, the patient would like to continue the eribulin as she felt better since the treatment. Therefore, we planned to continue this modified eribulin regime until the disease progression or limiting toxicities affected the patient's quality of life and activity of daily living (ADL).

\section{DISCUSSION}

Radiotherapy to the orbit produces rapid and satisfactory outcomes in choroidal metastases of breast cancer patients regardless of the underlying hormonal status [6]. A complete resolution of vision has been reported in up to $70 \%$ of patients following irradiation [7]. Our patient experienced an improvement in her vision as early as 12 weeks after radiotherapy and complete resolution of her vision at eight months after radiotherapy. This correlates with literature findings that clinical response to external beam radiotherapy (EBRT) can be delayed between 1-3 months or more [3].

There is no consensus on an optimal radiotherapy regime for choroidal metastases [8], but we prefer a shorter hypofractionated regime of 20 Gy over 5 days. It is important that the radiotherapy course not be protracted as choroidal metastases are usually part of the disseminated systemic disease. Prolonged radiotherapy will delay the start of systemic treatment with patients at risk of uncontrolled progression of distant metastases at other sites. This is especially so in triple-negative breast cancer which is known for its rapid progression.

The use of CyberKnife or stereotactic radiosurgery/ radiotherapy in choroidal metastases has the advantage of delivering radiotherapy at a higher dose over a shorter duration of between 1 to 3 days. However, this treatment is only available at the specialized radiotherapy center and is quite costly. Furthermore, an unwanted side effect of central retinal vein occlusion has been reported six months after radiosurgery [8].

Even though our center can offer stereotactic radiotherapy, we prefer conventional external beam radiotherapy (EBRT) with $6 \mathrm{MV}$ linac of laterally opposed beams. Our patient tolerated treatment well with no reported acute and late toxicity at nearly four years post-treatment.

Earlier case series reported a $12 \%$-rate of toxicity with conventional EBRT [9], but a recent case series reported no late toxicity and no grade-2-3 acute toxicity 
with this approach [10]. This difference is perhaps due to a shorter follow-up period in the later publication although the advancement and refinement of radiotherapy techniques may have been an important contributory factor as well.

Choroidal metastases in HER2-positive breast cancer may show a remarkable response with upfront trastuzumab [11], but no systemic agent can produce a similar response when used as an upfront treatment in triple-negative breast cancer. Therefore, in triplenegative breast cancer patients who are not in visceral crises, we prefer an upfront short course of palliative radiotherapy followed by an effective systemic therapy for a prolonged durable response.

Our patient had anthracycline and docetaxel chemotherapy in the past. We chose eribulin as the next line of treatment for our patient based on the landmark phase III multicenter randomized control trial which showed improvement of the overall survival when eribulin was used in patients who failed anthracycline and taxane-based chemotherapy [12]. The benefit is most significant in triple-negative patients with a median survival reported as 12.9 months compared to 8.2 months in the controlled arm [13] although its role, specifically in choroidal metastases, is not well established.

We found one case report of the use of eribulin in choroidal metastases as the eighth line of treatment in a HER2 amplified breast cancer patient. Eribulin gave the patient a complete resolution of choroidal metastases, but the response was only sustained for 12 months before the disease started to progress in the liver [14].

Further literature review failed to discover any reported case of eribulin used in choroidal metastases in triple-negative breast cancer. We believe that our case is the first reported.

Eribulin is a new generation systemic treatment with milder toxicity profiles compared to chemotherapy and can be used until the disease progression or limiting side effects $[12,13]$. The longest documented patient tolerated eribulin up to 65 cycles though the detail of the patient was not certain [15]. Our patient had managed 49 cycles of eribulin so far.

Although eribulin has successfully prolonged our patient's life beyond what is normally expected in triplenegative breast cancer, it is by far no magic bullet. While her disease is under control, we are now left to manage toxicities associated with prolonged exposure to eribulin although all the side effects are not different from those reported in major studies.

The most common side effect of eribulin reported by our patient was afebrile neutropenia which concurred with that reported in major clinical trials $[12,15]$. Our patient also experienced grade-I neuropathy which is also one of the other common side effects reported with the use of eribulin in other studies $[12,16]$. Other side effects of eribulin reported include fatigue, alopecia, and anemia. These were not seen in our patients despite the prolonged use of the drug.

Meanwhile, in major trials, reasons for drug discontinuation differ between trials. For example, Kauffman et al. [15] found that neutropenia as the most common adverse event led to discontinuation of eribulin while in a pooled analysis of two studies, Twelves et al. [13] found that peripheral neuropathy was the major cause.

Admitted by developing countries such as Malaysia, eribulin is considered expensive and, therefore, not accessible to most patients. Our patient is one of the fortunate who can receive the drug under federal government funding.

\section{CONCLUSIONS}

Our patient demonstrated that by combining local palliative radiotherapy and utilizing the latest systemic treatment available, it is possible to achieve prolonged survival in triple-negative breast cancer even those with choroidal metastases. Short course radiotherapy to the orbit, followed by continuous administration of eribulin, has resulted in nearly four years to the date of complete remission of choroidal metastases and prolonged survival of our patient.

\section{DECLARATIONS}

\section{Ethics of Approval Consent to Participate}

As this is a case report, ethics approval from the ethics committee is not required. We have received both verbal and written consent from the patient for permission to have her case written up including all images related to the case.

\section{Competing of Interest}

The authors declare no competing interest in this study.

\section{Acknowledgment}

None

\section{REFERENCES}

1. Azizah A, Hashimah B, Nirmal K, ET AL. Malaysian National Cancer Registry Report 2012-2016: Malaysia Cancer Statistic, Data and Figure [Internet]. Malaysia: National Cancer Institute; 2019 [cited 2021 October 30]. Available from: https://www.moh.gov. $\mathrm{my} / \mathrm{moh} /$ resources/Penerbitan/Laporan / Umum/2012-2016\%20(MNCRR)/MNCR_2012-2016_ FINAL_(PUBLISHED_2019).pdf

2. National Cancer Institute Ministry of Health Malaysia. Malaysian Study on Cancer Survival [Internet]. Putrajaya: National Cancer Institute Ministry of 
Health Malaysia; 2018 [cited 2021 October 30]. Available from: http://nci.moh.gov.my/index.php/ $\mathrm{ms} /$ main-menu-2/ laporan

3. Mathis $\mathrm{T}$, Jardel $\mathrm{P}$, Loria $\mathrm{O}$, et al. New concepts in the diagnosis and management of choroidal metastases. Prog Ret Eye Res. 2019;68:144-76.

4. Vlachostergios PJ, Voutsadakis IA, Papandreou CN. Orbital metastasis of breast carcinoma. Breast Cancer (Auckl). 2009;3:91-97.

5. Daoudi S, Touimi SH, Adrif I, et al. Ocular metastasis from breast cancer: case report. World J Innov Res. 2020;8(4):10-12.

6. Demirci H, Shields CL, Chao AN, Shields JA. Uveal metastasis from breast cancer in 264 patients. Am J Ophthalmology. 2003;136(2):264-71.

7. Mohammad J, Karthiga S, Khaled AQ, et al. Choroidal metastases from breast carcinoma: case series. Pan Arab J Oncol. 2011;4(1):6-10.

8. Cárdenas JRV, Penella ADV, Ibarra EC. Frameless radiosurgery for intraocular metastatic tumor: Case report. Rep Pract Oncol Radiother. 2020;25(1):1-5.

9. Rudoler SB, Corn BW, Shields CL, et al. External beam irradiation for choroid metastases: identification of factors predisposing to long-term sequelae. Int J Radiat Oncol Biol Phys. 1997;38(2):251-6.

10. Youbi ZA, Ciprian E, lonela $C$, et al. Management of choroidal metastasis using external beam radiotherapy: a retrospective study and review of the literature. J Cancer Metastasis Treat. 2017;3:105-10.
11. Wong ZW, Phillips SJ, Ellis MJ. Dramatic response of choroidal metastases from breast cancer to a combination of trastuzumab and vinorelbine. Breast J. 2004;10(1):54-6.

12. Cortes J, O'Shaughnessy J, Loesch D, et al. Eribulin monotherapy versus treatment of physician's choice in patients with metastatic breast cancer (EMBRACE): a phase 3 open-label randomised study. The Lancet. 2011;377(9769):914-23.

13. Twelves C, Cortes J, Vahdat L, et al. Efficacy of eribulin in women with metastatic breast cancer: a pooled analysis of two phase 3 studies. Breast Cancer Res Treat. 2014;148(3):553-61.

14. Ricciardi GR, Proto C, Ferraro G, Adamo V. Uncommon breast metastatic site and eribulin responsiveness in a heavily pretreated patient. Future Oncol. 2014;10(15):2417-22.

15. Kaufman PA, Awada A, Twelves C, et al. Phase III open-label randomized study of eribulin mesylate versus capecitabine in patients with locally advanced or metastatic breast cancer previously treated with an anthracycline and a taxane. J Clin Oncol. 2015;33(6):594-601.

16. Sirvén $M B$, Fernández-Ortega $A$, Stradella $A$, et al. Real-world efficacy and safety of eribulin in advanced and pretreated HER2-negative breast cancer in a Spanish comprehensive cancer center BMC Pharmacol Toxicol. 2019;20(1):1-7. 